\title{
The Added Value of MRI in Diagnosis of Cerebello-pontine Angle Tumors
}

\author{
Ahmed F. youssef , Ahmed E. Shaalan, Randa S. Moussa
}

\begin{abstract}
Department of Radiodiagnosis, Faculty of Medicine, Benha University hospitals, , Egypt

Correspondence to: Randa S. Moussa Department of Radiodiagnosis, Faculty of Medicine, Benha University hospitals, Egypt
\end{abstract}

\section{Email:}

randasaberbenha@gmail.com

Received: 15 April 2020

Accepted: 9 February 2021

\begin{abstract}
:
Background; Cerebello pontine angel masses are the most common neoplasm in posterior fossa accounting for 5-10\% of intracranial tumors. Aim; to evaluate the lesions that arise from this angle by using MRI sequences as T1 (with out and with contrast), T2, FLAIR, Diffusion Weighted and spectroscopy. Methods; This study included 25 patients age ranged 25 -55 years, and were subjected to full history talking, Clinical examination, laboratory investigations and MRI for patient with suspected cerebello pontine angle lesions. Results; Females are frequently involved by these lesions (19 patients) while males are less (6 patient). Among our patients, the most common presenting symptoms were hearing loss in 17 patients $(68.0 \%)$ headache in 10 patients $(40.0 \%)$, tinnitus in 9 patient ( 36.0 $\%$ ) neuralgia in 5 patients ( $20.0 \%$ ), vertigo in 2 patients $(8.0 \%)$, hemi facial spasm in one patient (4.0\%) and wasting of mastication muscles (4.0\%). Out of the studied patients, final diagnosis by MRI revealed acoustic schwannoma in 12 patients (48.0\%) which was the most common pathology, followed meningioma that were noted in 7
\end{abstract} patients $(28.0 \%)$, epidermoid cyst in one patient (4.0\%),prominent AICA in one patient (4.0 $\%)$, paraganglioma in one patient (4.0\%),cystic neurofibroma in one patient $(4.0 \%)$, neurofibromatosis type 2 in one patient $(4.0 \%)$ and arachnoid cyst in one patient (4.0\%). Conclusion: MRI is the gold standard method to differentiate the lesions by anatomical site of origin, shape, signal intensity and behavior after contrast media injection.

Keywords: MRI; Cerebello pontine angle; tumors 



\section{Introduction}

Cerebello pontine angle (CPA) masses are the most common neoplasm in posterior fossa accounting for 5-10\% of intracranial tumors (1).

The CPA cistern is a triangular space filled with cerebrospinal fluid. The lateral border is formed by the petrous temporal bone, at the medial side is the pons and at the posterior side is the cerebellar hemisphere. It extends into the internal auditory canal around the seventh (facial) and eighth (vestibulocochlear) cranial nerves. A large variety of the lesions that radiologists will encounter appear more and more frequently because of the remarkable sensitivity and accuracy of MRI imaging. (2)

The CPA cistern can be divided into anterior, posterior, superior and inferior regions by the plane of IAC. In the axial plane, the IAC divides the CPA into anterior and posterior compartment. The anterior compartment is contiguous with Pre-pontine cistern and contains cranial nerves (IV -VI) and branches of superior cerebellar, anterior inferior cerebellar and posterior inferior cerebellar arteries. The posterior compartment contains lateral lobes of the cerebellum and distal branches of a fore mentioned arteries. The superior compartment lies above the plan of IAC up to the level the tentorium cerebelli. The inferior compartment contains cranial nerves (IX - XII) (3).

Tumors of cerebellopontine angle may be asymptomatic or may present with a constellation of symptoms which could be due to tumor mass and involvement of the adjacent cranial nerves, cerebellum and brainstem. Furthermore, there could be associated signs and symptoms due to raised intracranial pressure (ICP) due to regional brain stem compression and hydrocephalus. the most common symptoms of CPA tumors are unilateral sensory neural hearing loss (96\%), un steadiness $(77 \%)$, tinnitus $(71 \%)$, headache (29\%), mastoid pain or otalgia (28\%), facial numbness (7\%) and diplopia (7\%) (4). Acoustic schwannoma is the most common tumor of CPA (80-90 \%) followed by meningioma (5-10 \%) ectodermal inclusion tumors, epidermoid and dermoid (5-7\%).other tumors of CPA include primary cholesteatoma and other cranial nerve schwannoma (V ,VII ,IX „X ), arachnoid cysts etc. (4).

Surgical approaches to the CPA vary depending on the tumor size, location and 
pre-operative neurologic function of the patient. The retro sigmoid approach, affording excellent visualization for large tumors and allowing for hearing preservation (5).

In recent years, advances in neuroradiology have facilitated the preoperative differentiation of CPA lesions and have aided the surgical planning (6).

Since introduction of magnetic resonance (MR), malformations of the brain have been found with greater frequency than previously suspected. This is especially true for any lesions in the cerebello pontine angle, where the sensitivity and the specificity of MR imaging with its multidimensional imaging capabilities are far superior to that of CT (7).

Computed tomography and magnetic resonance (MRI) are considered to be the conventional imaging modalities in the investigation of brain abnormalities .MRI having superior spatial and soft tissue contrast resolution has been established as the preferred method, further more by modifying the imaging parameters of scanning, MRI can address a variety of different diagnostic issues. MR images can be obtained isotopically at $1 \mathrm{~mm}$ intervals and can be reconstructed in three dimensions without loss of resolution .such post contrast scans are also suitable to measure tumor volume which is the newest method to evaluate tumor size before and after treatment ,replacing linear measurement (8).

The aim of our study was to evaluate the added value of MRI in diagnosis of cerebello pontine angle tumors.

\section{Patients and methods}

The study was done on 25 patients 6 males and 19 females their ages ranged from 2555 years old between 2019 and 2020. These patients were referred to the radio diagnosis department, MRI unit of Banha University from the ENT, neurology, and neurosurgery departments.

The patients present with the following symptoms sensoneuroral hearing loss in 17 patients, tinnitus in 8 patients, headache in 12 patients, vertigo in 3 patients, hemi facial spam in one patient, neuralgia in 5 patients and wasting of the muscles of mastication in one patient.

\section{Inclusion criteria:}

This study was conducted for patients with clinically suspicious cerebello pontine angle lesions mainly complaining of hearing loss, headache, tinnitus, vertigo, 
hemi facial spasm and wasting of muscles of mastication.

\section{Exclusion criteria:}

Patient having history of claustrophobia. Patient having history of metallic implants insertion, cardiac pacemakers. Metallic foreign body in situ. Patient clinically unstable. Trauma. Infectious pathology.

\section{All patients will be subjected to}

- Full history talking.

- Clinical examination.

- Laboratory investigation.

- MRI for patient with suspected cerebello pontine angle lesions.

Every patient was checked first for any MRI contraindication e.g cardiac pacemakers, aneurysmal clips for the patient needed anesthesia, fasting four hours before scan is required explanation of the procedure to patient for reassurance

MR brain with 1, 5 T MR Scanner

\section{Technique}

The study was performed while patients lying supine with their median sagittal plane perpendicular and coinciding with the midline of the scanner table with standard circulary polarized head coil

\section{1- Conventional MRI:}

Using sequences as axial $\mathrm{T} 1 \mathrm{~W}$, axial $\mathrm{T} 2$, axial FLAIR, coronal T2 and sagittal T1W without contrast, different pulse sequences and their examination times, in table 1

Table 1: Showing different pulse sequences and their examination times

\begin{tabular}{llllll}
\hline parameter & Axial T1 & Axial T2 & Axial Flair & Sagittal T1 & Coronal T2 \\
\hline TR & $485 \mathrm{msec}$ & $4400 \mathrm{msec}$ & $7200 \mathrm{msec}$ & $450 \mathrm{msec}$ & 4300 \\
TE & $12 \mathrm{msec}$ & $105 \mathrm{msec}$ & $100 \mathrm{msec}$ & $17 \mathrm{msec}$ & $105 \mathrm{msec}$ \\
FOV & 24 & 24 & 24 & 25.6 & 24 \\
MTX & $208 \times 352$ & $256 \times 448$ & 304 & $208 \times 352$ & $256 \times 448$ \\
NAQ & 1.0 & 1.0 & 1.0 & 1.0 & 1.0 \\
TIME & $2: 52$ & $1: 25$ & $3: 29$ & $2: 16$ & 00.48 \\
\hline
\end{tabular}

\section{2- Post contrast MRI :}

After intravenous administration of Gadolinium -DPTA in a dose of ,1-,,2 $\mathrm{mmol} / \mathrm{kg}$ body weight to maximum dose of $20 \mathrm{ml}$ through IV line within one minute .the examination started immediately at the end of injection with time ranged from 1215 minute according to protocol used .contrast enhanced in T1WI IN axial ,sagittal and coronal planes are obtained . 



\section{3- MR diffusion imaging :}

Three patients were examined by diffusion weighted image MRI sequences as they have doubtable diagnosis by conventional MRI sequences. Axial DW imaging was performed by using a single-shot T2weighted echo planar spin-echo sequences with the following parameters :1600/107,b values of 500 and $1000 \mathrm{sec} . \mathrm{mm} 2$; field of view, $24 \times 24 \mathrm{~cm}$; matrix size , $128 \times 128$; section thickness $7.5 \mathrm{~mm}$ section gap ,0 $\mathrm{mm}$; and one signal acquired. DW imaging was calculated and performed automatically by the MR instrument.

\section{4- Spectroscopic data analysis :}

MR spectroscopy was done in two patient of doubtable diagnosis the time versus signal intensity was processed to remove residual water signal . post processing of the spectroscopic data consisted of frequency shift, phase and linear base line correlation after FOURIER transformation . frequency domain curve was fitted to Gaussian line shape by using software provided by manufacturer to define NAA at 2,02 ppm ,choline at 3,02 $\mathrm{ppm} \mathrm{Cr}$ at 3,01 and lactate at $1,33 \mathrm{ppm}$.metabolic values were calculated automatically from the area under the metabolic peak using the standard commercial software program provided by the manufacturer. Peak integral values were normalized to the internal $\mathrm{Cr}$ peak .metabolite ratios of Choline /NAA NAA/CHO, NAA/Cr , $\mathrm{NAA} / \mathrm{Cr}+$ cho, cho /cr were calculated.

\section{Statistical analysis}

Data were fed to the computer and analyzed using IBM SPSS software package version 20.0. (Armonk, NY: IBM Corp) Qualitative data were described using number and percent. Our study revealed that schwannoma and meningioma are the most common lesions that arise in CPA and by MRI sequences, we can differentiate of them as $\mathrm{P}$ values is significant in MRI with contrast and association finding are 0,047 and less than 0,001 and with no significant $P$ values in T1 Weighted images or T2 weighted image without contrast 4333-692, all these finding are illustrated in detail latter.

\section{Results}

This study included 25 patients .Females were most commonly affected than males as 19 females (76\%) and males were 6 patients (24\%). figure (1).

The most age group frequently involved were 25-35 years (40\%) then age group 3545 years (32\%) and lastly age group 45-55 
years (28\%). This illustrated in the

following table (2).

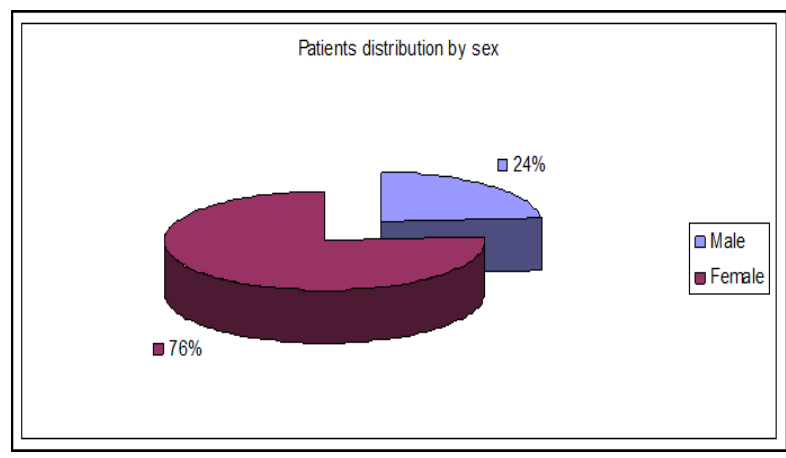

Fig (1): Patient's distribution by sex

Table (2): Patient's distribution by age

\begin{tabular}{lcc}
\hline & $\begin{array}{c}\text { Patients } \\
\text { distribution by age }\end{array}$ & Percent \\
\hline 25-35 years & 10 & $40 \%$ \\
35-45 years & 8 & $32 \%$ \\
$\mathbf{4 5 - 5 5}$ years & 7 & $28 \%$ \\
\hline
\end{tabular}

The clinical presentation of the 25 patients showed that the most common presenting symptoms were hearing loss in 17 patients (68.0\%), headache in10 patients $(40.0 \%)$, tinnitus in 9 patients $(36.0 \%)$, neuralgia in 5 patients $(20.0 \%)$, vertigo in 2 patients $(8.0 \%)$, hemifacial spasm in one patient $(4.0 \%)$ and wasting muscle of mastication in one patient (4.0\%).the patients may present with one or more of clinical symptoms, table (3).
Table (3): Clinical presentation of patients

\begin{tabular}{lcc}
\hline symptoms & number & percentage \\
\hline Hearing loss & 17 & $68.0 \%$ \\
Tinnitus & 9 & $36.0 \%$ \\
Headache & 11 & $44.0 \%$ \\
Vertigo & 2 & $8.0 \%$ \\
Hemifacial spasm & 1 & $4.0 \%$ \\
Wasting muscle of & 1 & $4.0 \%$ \\
mastication & & \\
Neuralgia & 5 & $20.0 \%$ \\
\end{tabular}

Lesions appear in MRI with different signals: in T1 weighted image isointense in 20 patients (80.0\%),hypointense in 4 patients $(16.0 \%)$ and hyperintense in one patient $(4.0 \%)$. while in T2 weighted image isointense in 14 patients (56.0\%), hyperintense in 10 patients $(40.0 \%)$ and hypointense in one patient $(4.0 \%)$. Table 4

Table (4): MRI signals (precontrast) of the lesions:

\begin{tabular}{llcc}
\hline & & number & percentage \\
\hline \multirow{3}{*}{ T1 } & Hypointense & 4 & $16.0 \%$ \\
& Isointense & 20 & $80.0 \%$ \\
& Hyperintense & 1 & $4.0 \%$ \\
& Hypointense & 1 & $4.0 \%$ \\
T2 & Isointense & 14 & $56.0 \%$ \\
& Hyperintense & 10 & $40.0 \%$ \\
\hline
\end{tabular}

Lesions appear with different pattern after contrast administration as: homogenous enhancement in 16 patients (64.0\%), heterogeneous enhancement in 6 patients (24.0\%),wall enhancement in one patient $(4.0 \%)$ and no enhancement in 2 patients $(8.0 \%)$. figure (2). 


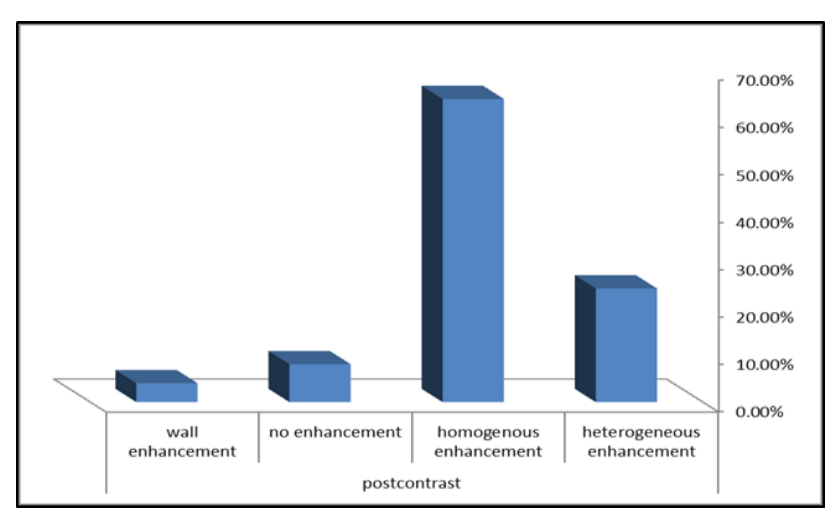

Fig (2): MRI pattern post-contrast of the lesions

There are some associations finding in MRI helping in diagnosis as: extension into internal auditory canal in 8 patients (32.0\%), dural tail in 5 patients $(20.0 \%)$, dural base in 2 patients $(8.0 \%)$,extension from skull base in one patient $(4.0 \%)$ and no associations in 2 patients $(8.0 \%)$. Figure (3).

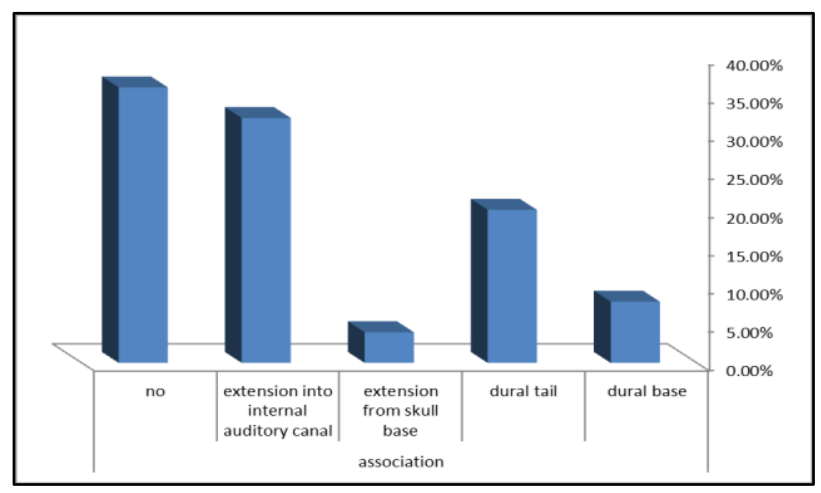

Fig (3): Association finding in MRI

Out of the studied 25 patients, final diagnosis by MRI revealed acoustic schwannoma in 12 patients $(48.0 \%)$ which was the most common pathology, followed meningioma that were noted in 7 patients $(28,0 \%)$, epidermoid cyst in one patient
(4.0\%) ,prominent AICA in one patient $(4.0 \%)$, paragangilioma in one patient (4.0\%),cystic neurofibroma in one patient (4.0\%), neurofibromatosis type 2 in one patient $(4.0 \%)$ and arachnoid cyst in one patient (4.0\%). figure (4).

Comparison between acoustic schwannoma and meningioma revealed by $\mathrm{T} 1$ weighted images as hypointense in one patient of acoustic shawannoma $(8.3 \%)$ but meningioma has $\operatorname{not}(0.0 \%)$, isointense in 11 patients of acoustic schwannoma $(91.7 \%)$ but meningioma in 7 patients $(100 \%)$ and hyperintense in no patient of both acoustic shawannoma and meningioma $(0.0 \%)$. In $\mathrm{T} 2$ weighted images it was hypointense in one patient of acoustic shawannoma ( $8.3 \%)$ but no patient in meningioma $(0.0 \%)$, isointense in 7 patients of acoustic shawannoma (58.3\%) and 5 patients in meningioma (71.4\%) and hyperintense in 4 patients of acoustic shawannoma $(33.3 \%)$ but 2 patients of meningioma $(28.6 \%)$. All these illustrated data has no significant $\mathrm{P}$ value (.433 and .692). Table (5).

Comparison between acoustic shawannoma and meningioma by signal intensity after contrast as heterogeneous enhancement in 5 patients of acoustic shawannoma (41.7\%) and no patient in meningioma and 
homogenous enhancement in 7 patients of acoustic shawnnoma (58.3\%) but it was in 7 patients of meningioma (100\%) It has significant $\mathrm{P}$ value (0.047). Table (6).

has not $(0.0 \%)$.Extension into internal auditory canal in 8 patients of acoustic schwannoma but meningioma has not. There is no associated MRI finding in 4
Comparison between acoustic schwannoma and meningioma by associated MRI finding as lesion has dural base in 2 patients $(28.6 \%)$,dural tail in 5 patients $(71.4 \%)$ in meningioma while acoustic s schwannoma patients of acoustic schwannoma (33.3\%). It has significant $\mathrm{P}$ value $(<0.001)$. Table (7).

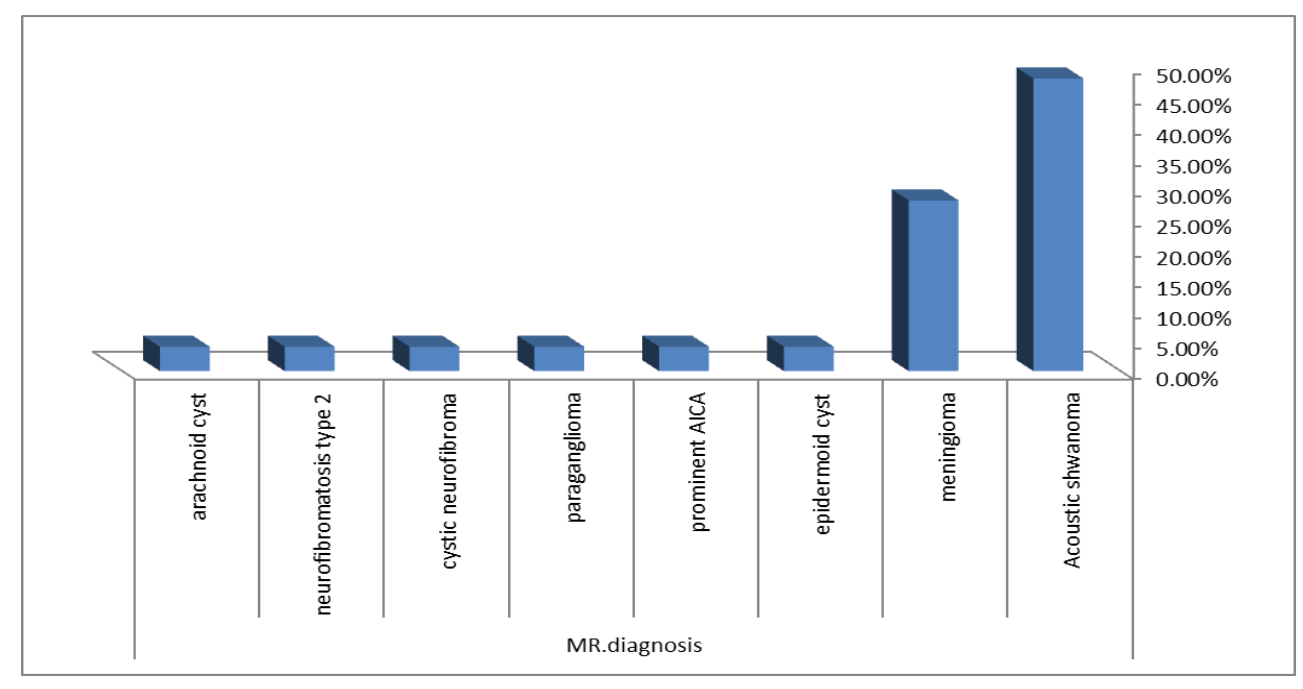

Fig (4): MRI diagnosis

Table (5): Comparison of schwannoma and meningioma by MRI T1, T2 pre-contrast

\begin{tabular}{|c|c|c|c|c|c|c|c|}
\hline & & \multicolumn{4}{|c|}{ MR diagnosis } & \multirow[t]{3}{*}{$X^{2}$} & \multirow[t]{3}{*}{ p-value } \\
\hline & & \multicolumn{2}{|c|}{ Acoustic schwannoma } & \multicolumn{2}{|c|}{ Meningioma } & & \\
\hline & & No. & $\%$ & No. & $\%$ & & \\
\hline \multirow{3}{*}{ T1 } & Hypointense & 1 & $8.3 \%$ & 0 & $0.0 \%$ & .616 & .433 \\
\hline & Isointense & 11 & $91.7 \%$ & 7 & $100.0 \%$ & & \\
\hline & Hyperintense & 0 & $0.0 \%$ & 0 & $0.0 \%$ & & \\
\hline \multirow{3}{*}{$\mathbf{T} 2$} & Hypointense & 1 & $8.3 \%$ & 0 & $0.0 \%$ & .735 & 692 \\
\hline & Isointense & 7 & $58.3 \%$ & 5 & $71.4 \%$ & & \\
\hline & Hyperintense & 4 & $33.3 \%$ & 2 & $28.6 \%$ & & \\
\hline
\end{tabular}


Table (6): Comparison of schwannoma and meningioma by MRI postcontrast:

\begin{tabular}{llcccccc}
\hline & \multicolumn{5}{c}{ MR diagnosis } & $\mathbf{X}^{2}$ & p-value \\
& \multicolumn{2}{c}{ Acoustic schwannoma } & \multicolumn{2}{c}{ Meningioma } \\
& No. & $\%$ & No. & $\%$ & \\
\hline Post-contrast & $\begin{array}{l}\text { Heterogeneous } \\
\text { enhancement } \\
\text { homogeneous } \\
\text { enhancement }\end{array}$ & 5 & $41.7 \%$ & 0 & $0.0 \%$ & $\mathbf{5 . 1 1 5}$ & $\mathbf{. 0 4 7 ^ { * }}$ \\
\hline
\end{tabular}

Table (7): Comparison between schwannoma and meningioma by MRI associated finding:

\begin{tabular}{|c|c|c|c|c|c|c|c|}
\hline & & \multicolumn{4}{|c|}{ MR diagnosis } & \multirow[t]{3}{*}{$\mathbf{X}^{2}$} & \multirow[t]{3}{*}{ p-value } \\
\hline & & \multicolumn{2}{|c|}{ Acoustic schwannoma } & \multicolumn{2}{|c|}{ Meningioma } & & \\
\hline & & No. & $\%$ & No. & $\%$ & & \\
\hline \multirow{4}{*}{ Association } & Dural base & 0 & $0.0 \%$ & 2 & $28.6 \%$ & \multirow[t]{4}{*}{19.00} & \multirow[t]{4}{*}{$<0.001 * *$} \\
\hline & Dural tail & 0 & $0.0 \%$ & 5 & $71.4 \%$ & & \\
\hline & Extension into internal & 8 & $66.7 \%$ & 0 & $0.0 \%$ & & \\
\hline & no & 4 & $33.3 \%$ & 0 & $0.0 \%$ & & \\
\hline
\end{tabular}

\section{Discussion}

The patients of this study represented arrange of of age group from $25-55$ years with the mean $\mathrm{SD} \pm 39.9$. The most age group involved were 25-35 years old, by $(40 \%)$, this is going some studies (9).

The relationship of sex and the lesions arise in cerebello pontine angle revealed that females are commonly affected (76\%) , while males less commonly affected ( $24 \%$ ) which is not going with the previous study (9).

Patients of CPA lesions suffer from some symptoms, the most common presenting symptoms were hearing loss (68\%), headache (40\%), tinnitus (36\%), neurolagia $(20 \%)$, vertigo (8\%) and lastly hemifacial spasm and wasting muscles of mastication (4\%) for each of them, that is going with the study done in 2000 (10).

The final diagnosis by MRI of cerebello pontine angle revealed that vestibular schwannoma $(48.0 \%)$ was the most common pathology, followed by meningioma $(28.0 \%)$, epidermoid cyst (4.0 \%), prominent AICA (4.0\%), paraganglioma $\quad(4.0 \%), \quad$ Cystic neurofibroma (4.0\%), neurofibromatosis type $2(4.0 \%)$. It was reported, in another research (6) that the most common 
cerebello pontine angle tumor is vestibular schwannomas that accounts for about $80 \%$ followed by Meningioma $10 \%$ then epidermoid cyst $6 \%$

In our study schwannoma revealed by $\mathrm{T} 1$ weighted images as iso intense (91.7\%), hypo intense $(8.3 \%)$ and in $\mathrm{T} 2$ weighted image it was hyper intense $(33.3 \%)$, iso intense (58.3\%) and hypo intense (8.3\%), Signal intensity after contrast is heterogeneous enhancement ( $41.7 \%)$ and homogenous enhancement ( $58.3 \%$ ).

Durai , et al (11) reported that Schwannomas on MRI are isointense or mildly hypo intense on T1WI and mildly hyper intense on T2WI with variable enhancement. They maybe heterogeneous due to necrosis, hemorrhagic components, and occasional calcification. Widening of internal auditory canal is an important finding .(11)

In our study schwannoma appear relative in diffusion DWI, unless there is restriction in diffusion as epidermoid cyst that was also reported before (12).

MR spectroscopy of schwannoma is done in some patients of our study because these patients have past history of cancer breast with doubtful metastasis, we found myoinositol and choline peak elevation and with no elevation of lipid and choline which are characteristic of metastasis, That is going hand in hand with other studies (12).

In our study comparison between acoustic schwannoma and meningioma revealed by T1 weighted images as hypo intense of acoustic schwannoma $8.3 \%$, But meningioma has not $(0.0 \%)$, isointense of acoustic schwannoma ( 91.7\%) but meningioma $100 \%$ and hyper intense in no patient of both acoustic schwannoma and meningioma( $0.0 \%$ ). In $\mathrm{T} 2$ weighted images it was hypo intense of acoustic schwannoma $(8.3 \%)$ but no patient in meningioma ( $0.0 \%)$, isointense acoustic schwannoma( $58.3 \%$ ) and meningioma ( $71.4 \%)$ and hyper intense of acoustic schwannoma ( $33.3 \%$ ) but meningioma( $28.6 \%$ )

In our study comparison between vestibular schwannoma and meningioma by associated MRI finding as lesion has dural base $(28.6 \%)$, dural tail $(71.4 \%)$ in meningioma while vestibular schwannoma has not $(0.0 \%)$. Extension into the internal auditory canal happens in vestibular schwannoma but not in meningioma.

As reported by Yadav., et al (13) Meningioma involving the dura of the 
posterior margin of the petrous temporal bone can project posteriorly into the CPA and simulate a vestibular schwannoma. These two neoplasms may have identical signal intensity characteristics on MRI. However, meningiomas are usually situated eccentric to the IAC and form a more obtuse angle with the petrous ridge than schwannomas. Extension of meningioma into the IAC is uncommon but not unknown, and rarely schwannoma may simulate the appearance of a dural tail on post contrast images .

In our study epidermoid cyst in CPA show hypo intense in T1 weighted images, hyper intense in T2 weighted and heterogeneous intensity on flair image. It shows also restricted diffusion ( high signal) with no enhancement after contrast administration.

As reported by Caballeros., et al (2) epidermoids may insinuate themselves around normal vascular structures rather than displace them. On MRI, Epidermoid Cysts are heterogeneously hyper intense on both $\mathrm{T} 1$ and $\mathrm{T} 2$ weighted sequences, they are restrictive on DWI and do not enhance after contrast administration. It is important to note that they do not suppress on FLAIR sequences.
In our study arachnoid cyst appear as hypo intense in $\mathrm{T} 1$ weighted image, hyper intense T2 weighted image and hypo intense in DW image. It has no enhancement after contrast injection

As reported by Farhan., et al (14); it was easy to differentiate between epidermoid cyst and arachnoid cyst on diffusionweighted image; this differentiation was difficult on conventional MRI sequences even after IV contrast. The epidermoid cyst was the only lesion that appears brightly hyper intense on DW.

\section{Conclusion}

Many imaging modalities are used to differentiate the lesions arising from CPA including CT, MRI, PET CT and Angiography. CT is the most available method for diagnosis of cerebellopontine angle lesions, but MRI is the gold standard method to differentiate the lesions by anatomical site of origin, shape, signal intensity and behavior after contrast media injection .some cases need complementary advanced MRI technique as diffusion weighted image and spectroscopy.

\section{References}

1. Mersha HB, Kebebew $\mathrm{T}$, Debebe $\mathrm{T}$. Cerebellopntine angle masses: RadiologicPathology correlation at Tikur Anbessa 
Benha medical journal vol. 38, special issue (Radiology), 2021

Specialized Hospital and Myungsung Christian Medical Center . Ethiop Med J. 2018;56(1).

2. Caballeros F, Madrid JM, Bartolomé P, Domínguez $\mathrm{P}$, de Eulate $\mathrm{MRG}$, Zubieta J. Cerebellopontine angle masses: an MRI review for the resident. In European Congress of Radiology 2016; 2016.

3. Sayyahmelli S, Leonhard L, Ozaydin B, Roche JP. Vestibular Schwannomas: Neurology, Neurophysiology and Anatomy. In: Vestibular Schwannoma Surgery. Springer; 2019. p. 1558.

4. Memari F, Hassannia F, Abtahi SHR. Surgical outcomes of cerebellopontine angle tumors in 50 cases. Iran J Otorhinolaryngol. 2015;27(78):29.

5. Heller RS, Silveira L, Heilman CB. Cerebellopontine Angle Tumors. In: Principles of Neurological Surgery. Elsevier; 2018. p. $622-31$.

6. Kankane VK, Warade AC, Misra BK. Nonvestibular schwannoma tumors in the cerebellopontine angle: A single-surgeon experience. Asian J Neurosurg. 2019;14(1):154.

7. Kapoor S, Kulkarni VM. Role of MRI in Evaluation of Cerebello pontine angle tumour. J Adv Med Dent Sci Res. 2018;6(9):15-8.

8. Gouliamos AD, Patronas NJ. Conventional imaging in the diagnosis of brain tumors. In:
Imaging in Clinical Oncology. Springer; 2018. p. $135-50$.

9. Asaoka K, Barrs DM, Sampson JH, McElveen JT, Tucci DL, Fukushima T. Intracanalicular meningioma mimicking vestibular schwannoma. Am J Neuroradiol. 2002;23(9):1493-6.

10. Walsh RM, Bath AP, Bance ML, Keller A, Rutka JA. Consequences to hearing during the conservative management of vestibular schwannomas. Laryngoscope. 2000;110(2):250.

11. Durai I, Ganesan P, Selvaganapathy A, Moorthy D, Premkumar G. Role Of MRI in evaluation of posterior fossa tumours with histopathological correlation. J Evol Med Dent Sci. 2016;5(68):4927-34.

12. Lakshmi M, Glastonbury CM. Imaging of the cerebellopontine angle. Neuroimaging Clin. 2009;19(3):393-406.

13. Yadav P, Jantre M, Thakkar D. Magnetic resonance imaging of cerebellopontine angle lesions. Med J Dr DY Patil Univ. 2015;8(6):751.

14. Farhan DA, Abdulsttar OA. Role of Diffusion-Weighted Image in Differentiation between Epidermoid Cyst and other Cerebellopontine Angle Masses. Med J Babylon. 2018;15(3):238-42.

To cite this article: Ahmed F. youssef, Ahmed E. Shaalan, Randa S. Moussa. The Added Value of MRI in Diagnosis of Cerebello-pontine Angle Tumors. BMFJ 2021;38 (Radiology): 174-185, DOI: $10.21608 / \mathrm{bmfj} .2020 .27981 .1249$ 